Optimizing long-range order, band gap, and group velocities for graphene on close-packed metal surfaces

This article has been downloaded from IOPscience. Please scroll down to see the full text article.

2012 J. Phys.: Condens. Matter 24314203

(http://iopscience.iop.org/0953-8984/24/31/314203)

View the table of contents for this issue, or go to the journal homepage for more

Download details:

IP Address: 128.178.174.191

The article was downloaded on 23/07/2012 at 09:05

Please note that terms and conditions apply. 


\title{
Optimizing long-range order, band gap, and group velocities for graphene on close-packed metal surfaces
}

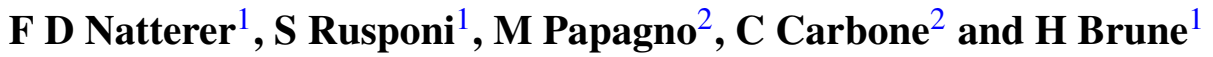 \\ ${ }^{1}$ Institute of Condensed Matter Physics (ICMP), Ecole Polytechnique Fédérale de Lausanne (EPFL), \\ CH-1015 Lausanne, Switzerland \\ ${ }^{2}$ Istituto di Struttura della Materia, Consiglio Nazionale delle Ricerche, Basovizza, Trieste, Italy
}

Received 24 December 2011, in final form 6 February 2012

Published 20 July 2012

Online at stacks.iop.org/JPhysCM/24/314203

\begin{abstract}
We compare different growth methods with the aim of optimizing the long-range order of a graphene layer grown on $\mathrm{Ru}(0001)$. Combining chemical vapor deposition with carbon loading and segregation of the surface layer leads to autocorrelation lengths of $240 \AA$. We present several routes to band gap and charge carrier mobility engineering for the example of graphene on $\operatorname{Ir}(111)$. Ir cluster superlattices self-assembled onto the graphene moiré pattern produce a strong renormalization of the electron group velocity close to the Dirac point, leading to highly anisotropic Dirac cones and the enlargement of the gap from 140 to $340 \mathrm{meV}$. This gap can further be enhanced to $740 \mathrm{meV}$ by Na co-adsorption onto the Ir cluster superlattice at room temperature. This value is close to that of $\mathrm{Ge}$, and the high group velocity of the charge carriers is fully preserved. We also present data for $\mathrm{Na}$ adsorbed without the Ir clusters. In both cases we find that the $\mathrm{Na}$ is on top of the graphene layer.
\end{abstract}

(Some figures may appear in colour only in the online journal)

\section{Introduction}

Graphene $(\mathrm{g})$ is a $2 \mathrm{D}$ honeycomb lattice made of carbon atoms with the conduction electrons in $\mathrm{sp}^{2}$-hybridized Bloch states. Its exceptional charge carrier mobility and optical transparency of $98 \%$ make graphene an ideal material for fast transistors [1], and for top electrodes for solar cells. These exciting and potentially useful properties of graphene are delicate functions of the structural and chemical defect density [2] and thus strongly depend on the quality of the graphene layer. On the other hand, for the creation and implementation of reliable devices, a reproducible graphene production process for large areal quantities is required [3-5]. A major effort is therefore devoted to simultaneously satisfying the two fundamental key issues: the production of defect free graphene and the scalability and transferability of the chosen preparation process. In this sense, albeit high quality, the popular method of mechanically exfoliating graphene sheets from highly oriented pyrolytic graphite with adhesive tape [6] has to be disregarded due to its impracticality beyond prototyping. The alternative and scalable approaches rely on a supporting substrate that catalyzes and seeds graphene growth. One often considered method consists in exposing an atomically clean single crystal surface to a hydrocarbon gas partial pressure and heat treatment to induce pyrolysis of the molecules in temperature programmed growth (TPG) or chemical vapor deposition (CVD). While low temperature chemisorption of hydrocarbon molecules is followed by flash annealing [7-14] in the first case, the latter approach involves high temperature exposure of hydrocarbon molecules to the catalytically active substrate [3-5, 15-18]. The pyrolysis of the hydrocarbon molecules enriches the substrate surface with carbon adatoms to finally yield graphene. Alternatively, surface segregation of bulk dissolved carbon impurities [18-23] or molecular beam epitaxy from high purity carbon rods at elevated temperatures [24] can also be employed. We discuss four of these growth techniques for the case of a $\mathrm{Ru}(0001)$ substrate [25] with special focus on the resulting long-range order, expressed in terms of the autocorrelation length that is a finger-print of the structural quality of graphene. 
A related, and for applications equally relevant, issue is the ability to engineer the graphene electronic properties. Close to the K-points of the Brillouin zone, the bands have a linear dispersion that is well described by the relativistic Dirac equation for massless neutrinos. The resulting Dirac cones of the conduction and valence bands touch each other at the Dirac point located at the Fermi level $E_{\mathrm{F}}$. Therefore free-standing graphene is a zero-gap semiconductor. However, most electronic device applications require a band gap. Theory has suggested that a gap may derive from an external long-range periodic potential $[13,26,27]$. Such a potential is created when adsorbing graphene onto a lattice mismatched close-packed metal surface. The resulting moiré structures exhibit periodic stacking alternations where either one of the two $\mathrm{C}$ atoms in the unit cell is localized on top of a metal atom, or both $\mathrm{C}$ atoms are adsorbed on substrate hollow sites. The binding of graphene to the substrate has a significant van der Waals contribution; however, in the on-top stacking areas a hybridization of graphene $\pi$ and metal $\mathrm{d}$ states can be established. This inhomogeneous binding induces a weak corrugation in the electron potential with the period of the moiré pattern which is manifested in a small band gap. This corrugation can be reinforced by adsorbing metal clusters on top. For Ir clusters on $\mathrm{g} / \mathrm{Ir}(111)$ an $\mathrm{sp}^{3}$ hybridization with the metal has been reported [28]. This re-hybridization is localized at the graphene areas covered by clusters, since only there can one of the two $\mathrm{C}$ atoms of the unit cell hybridize with a cluster Ir atom above and the other with a substrate Ir atom below. We show the experimental realization of this concept of band gap engineering for equidistant Ir clusters grown on $\mathrm{g} / \mathrm{Ir}(111)$ [29]. Alternative methods of inducing band gaps in graphene layers ranging from a few tens to a few hundreds of meV are patterning [30-33], adsorption [34-36] and intercalation of suitable elements [37, 38], strain [39] or substrate induced symmetry breaking $[40,41]$. However, most of these methods have the drawback of producing a flattening of the Dirac cone and thus a reduction of the electron group velocity, an effect which should be minimized for graphene applications in electronic circuits since a high group velocity is the necessary condition for high carrier mobilities. We further show the ability of tuning the graphene electronic band gap by adsorbing alkali metals on top of graphene [42]. Sodium adsorption on bare graphene $(\mathrm{Na} / \mathrm{g})$ and on a well ordered superlattice of Ir clusters on graphene $(\mathrm{Na} / \mathrm{Ir} / \mathrm{g})$ on $\operatorname{Ir}(111)$ produces very large band gaps while preserving the shape of the Dirac cone and thereby the high electron group velocity.

\section{Experiment}

The presented measurements were performed with a homemade scanning tunneling microscope (STM), operating at room temperature and at a base pressure below $5 \times 10^{-11}$ mbar [43]. All listed pressure values state the readout of a $\mathrm{N}_{2}$ calibrated cold-cathode gauge without correcting for the respective hydrocarbon or process gas sensitivity. The STM images were acquired in the constant current mode and with an electrochemically etched W tip.
The indicated tunnel voltages $V_{\mathrm{t}}$ correspond to the sample potential. Our $\mathrm{Ru}(0001)$ single crystal was cleaned by repeated cycles of $\mathrm{Ar}^{+}$ion sputtering (first $300 \mathrm{~K}, 30 \mathrm{~min}$, then $1030 \mathrm{~K}, 15 \mathrm{~min}, 1.5 \mu \mathrm{A} \mathrm{cm}{ }^{-2}, 1.2 \mathrm{kV}$ ), annealing in oxygen $\left(1030 \mathrm{~K}, 8 \mathrm{~min}, 8 \times 10^{-8} \mathrm{mbar}\right)$ and flash to $1650 \mathrm{~K}$. Cleanliness of the Ru surface was checked with STM and the stated cleaning routine repeated if required. The carbon source was either ethylene $\left(\mathrm{C}_{2} \mathrm{H}_{4}\right)$ introduced into the UHV system via a leak-valve or dissolved bulk carbon impurities as will be detailed later.

The $\operatorname{Ir}(111)$ crystal was prepared by repeated cycles of $\mathrm{Ar}^{+}$ion sputtering at room temperature and at $1000 \mathrm{~K}$, followed by annealing to $1500 \mathrm{~K}$. The graphene layer was grown by exposing the $\operatorname{Ir}(111)$ surface at $1300 \mathrm{~K}$ to ethylene [15, 16]. For the ethylene pressures and exposures used CVD growth is self-limiting and only a single graphene layer forms, which we confirmed by angle-resolved photoelectron spectroscopy (ARPES). The Ir cluster superlattice was grown by evaporating $0.15 \mathrm{ML} \mathrm{Ir}$ (one monolayer, ML, is defined as one atom per Ir substrate atom) at a substrate temperature of $(375 \pm 25) \mathrm{K}$. Under these conditions, exactly one Ir cluster with a mean cluster size of 13 atoms is formed per hcp region of the $\mathrm{g} / \mathrm{Ir}(111)-(9.32 \times 9.32)$ moiré $[12,16]$. Ir was evaporated from a current heated thin film plate with a cross section of $0.5 \mathrm{~mm} \times 0.1 \mathrm{~mm}$. The deposition flux was adjusted to $2.0 \times 10^{-4} \mathrm{ML} \mathrm{s}^{-1}$ and calibrated by the core level intensity measured for Ir deposition on a $\operatorname{Ag}(111)$ crystal. This calibration was cross-checked with STM in a different UHV chamber. Sodium was evaporated from a commercial getter source (SAES) onto the sample held at room temperature. ARPES data were collected at the VUV beam line of the ELETTRA synchrotron radiation facility in Trieste with a photon energy of $120 \mathrm{eV}$. This energy is close to the Cooper minimum of the photoionization cross section of the $\operatorname{Ir} 5 \mathrm{~d}$ states and thereby minimizes the Ir background photoemission signal with respect to the graphene states. ARPES spectra were recorded at a sample temperature of $100 \mathrm{~K}$ with a Scienta R-4000 hemispherical electron analyzer which allows spectra to be recorded simultaneously within an angular aperture of $30^{\circ}$.

\section{3. $g / \operatorname{Ru}(0001)$}

The chemisorption of a saturated monolayer (ML) of ethylene onto $\mathrm{Ru}(0001)$ at room temperature, followed by thermal dehydrogenation at $1100 \mathrm{~K}$, gives rise to the graphene islands visible on the atomic substrate terraces in figure 1(a). The periodic pattern resolved in the islands is the $(23 \times 23)$ moire structure [44-46] caused by the lattice mismatch between the $\mathrm{Ru}$ substrate and the graphene layer. On $\mathrm{Pt}(111)$, the chemisorption of a saturated layer of ethylene at $300 \mathrm{~K}$ leads, after pyrolysis, to a coverage of $0.25 \mathrm{ML}[8,10$, $47,48]$. On $\mathrm{Ru}(0001)$ we measured after one chemisorption and annealing cycle a graphene coverage of $\Theta=(0.23 \pm$ $0.05) \mathrm{ML}$, in agreement with this value. A second TPG cycle leads to a coverage of $(0.43 \pm 0.05) \mathrm{ML}$ and triggers coalescence of graphene islands, as seen in figure 1(b). 
(a)

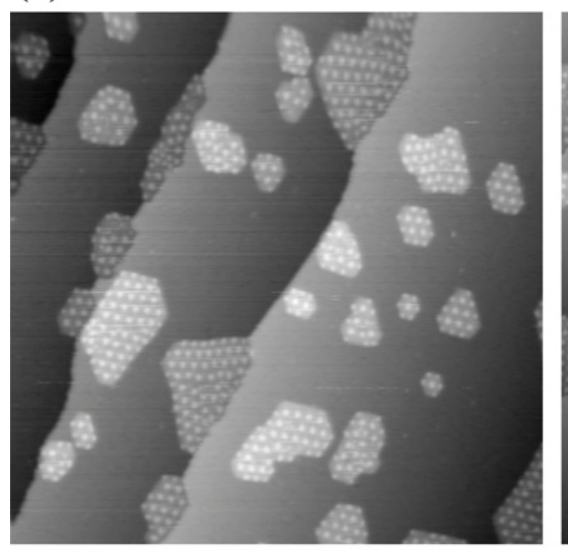

$300 \AA$ (b)

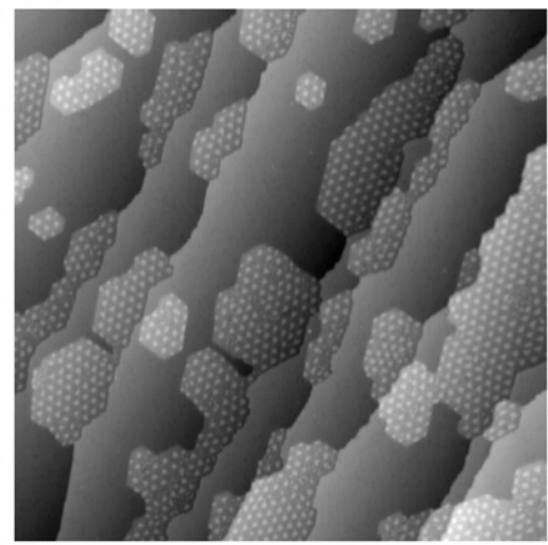

$300 \AA$

Figure 1. Graphene growth by chemisorption and dissociation cycles of ethylene on $\mathrm{Ru}(0001)$. (a) STM constant current image after the first cycle, leading to a g coverage of $\Theta=0.23 \mathrm{ML}$. (b) STM image after the second step, showing $\Theta=0.43$ ML (one cycle consists of exposure to $5 \mathrm{~L} \mathrm{C}_{2} \mathrm{H}_{4}$ at $T_{\text {ads }}=300 \mathrm{~K}$, dissociation and $\mathrm{H}_{2}$ desorption at $T_{\text {diss }}=1100 \mathrm{~K}$, STM parameters $V_{\mathrm{t}}=-1.0 \mathrm{~V}, I_{\mathrm{t}}=316 \mathrm{pA}$, $T=300 \mathrm{~K})$.

Since there are many translational domains of the moiré structure, island coalescence leads with a high probability to domain walls. In contrast to $\mathrm{g} / \mathrm{Ir}(111)$, where the domain walls can be removed by annealing [15], we observe that they stay on $\mathrm{Ru}(0001)$. This is attributed to the stronger binding of $\mathrm{g}$ to $\mathrm{Ru}(0001)$ [44, 49, 50] than to $\operatorname{Ir}(111)$ [51], where the weaker substrate interaction enables diffusion of entire islands in a Smoluchowski ripening process [15]. A third TPG cycle leads to $(0.54 \pm 0.05)$ ML. Therefore the coverage as a function of the number of cycles $n$ is described by $\Theta=1-(1-0.24)^{n}$. This is the expected behavior for selective ethylene dehydrogenation on the catalytically active transition metal surface, whereas graphene has a low sticking coefficient to hydrocarbons and a negligible dehydrogenation activity [17]. As a consequence, graphene growth by sequential chemisorption and decomposition has the advantage of being self-limiting. One asymptotically approaches a single layer graphene and no second layer will grow. However, it suffers from the many domain walls limiting the long-range order. For $\mathrm{Ru}(0001)$ these domain walls cannot be removed by annealing.

In CVD graphene growth the $\mathrm{Ru}(0001)$ surface is exposed to ethylene at $T_{\mathrm{CVD}} \geq 1000 \mathrm{~K}$. However, $\mathrm{C}$ dissolves into the $\mathrm{Ru}$ bulk, and, in addition, the solubility of interstitial carbon in ruthenium shows a strong temperature dependence-it is six times higher at $1540 \mathrm{~K}$ than at $1000 \mathrm{~K}$ [19]. In order to prevent diffusion into the bulk, as well as surface segregation of already dissolved C, CVD growth has to be performed at not too high a temperature and the sample temperature has to be rapidly quenched afterwards. Both requirements are somewhat in contradiction with best order. For the quench the best compromise is $\dot{T}=-7 \mathrm{~K} \mathrm{~s}^{-1}$, and the influence of the growth temperature is illustrated in figure 2 . In both cases there is a single monolayer of graphene and it is evident from visual inspection that the higher exposure temperature leads to better long-range order. This is quantified by determining the exponential decay lengths of the 2D autocorrelation functions ${ }^{3}$. We find an autocorrelation length of $\Lambda=(131 \pm$ 8) $\AA$ for $T_{\mathrm{CVD}}=1650 \mathrm{~K}$, while $T_{\mathrm{CVD}}=1100 \mathrm{~K}$ leads to $\Lambda=(68 \pm 12) \AA$. When disregarding the bulk solubility, CVD is also self-limiting since we find that growth of the second monolayer requires significantly higher exposures. The delicate issue with CVD growth is the bulk solubility at the temperature giving best order. This can be turned into an advantage, as shown below; however, it renders control on the graphene coverage more difficult.

The third growth method combines CVD surface growth with deliberate loading of the surface region with carbon which is then segregated by a very slow cool down [22]. By optimizing the parameters, we achieved very well ordered graphene layers, as seen in figure 3(a), with an autocorrelation length of $\Lambda=(238 \pm 12) \AA$. The superior long-range order is the most prominent argument for this technique. However, one needs to keep track of the coverage as multiple layers may be nucleated if sufficient interstitial carbon has been created before [52].

The fourth growth technique is the mere segregation of $\mathrm{Ru}$ bulk dissolved carbon impurities. The amount of $\mathrm{C}$ present in commercial $\mathrm{Ru}$ crystals suffices to create relatively well ordered graphene layers for a large number of experiments by a flash to $T=2000 \mathrm{~K}$ [20]. However, in comparison to the third method, one encounters the complication of co-segregation of elements other than $\mathrm{C}$, which may lead to structural imperfections in the graphene layer, such as point or substitutional defects [53]. After many sputter, oxygen glowing and flash cycles, the Ru becomes entirely clean [20] and then one may load it with only $\mathrm{C}$ in the way described above. When a sample is frequently used for CVD some of the $\mathrm{C}$ is dissolved and gradually accumulated in the bulk such

\footnotetext{
3 The spatial 2D autocorrelation is defined as $a c_{i, j}=\sum_{k, l=1}^{n} z_{k l} \cdot z_{k+i, l+j}$, i.e., it multiplies the pixels of the image translated by $i, k$ with the ones of the non-translated image.
} 

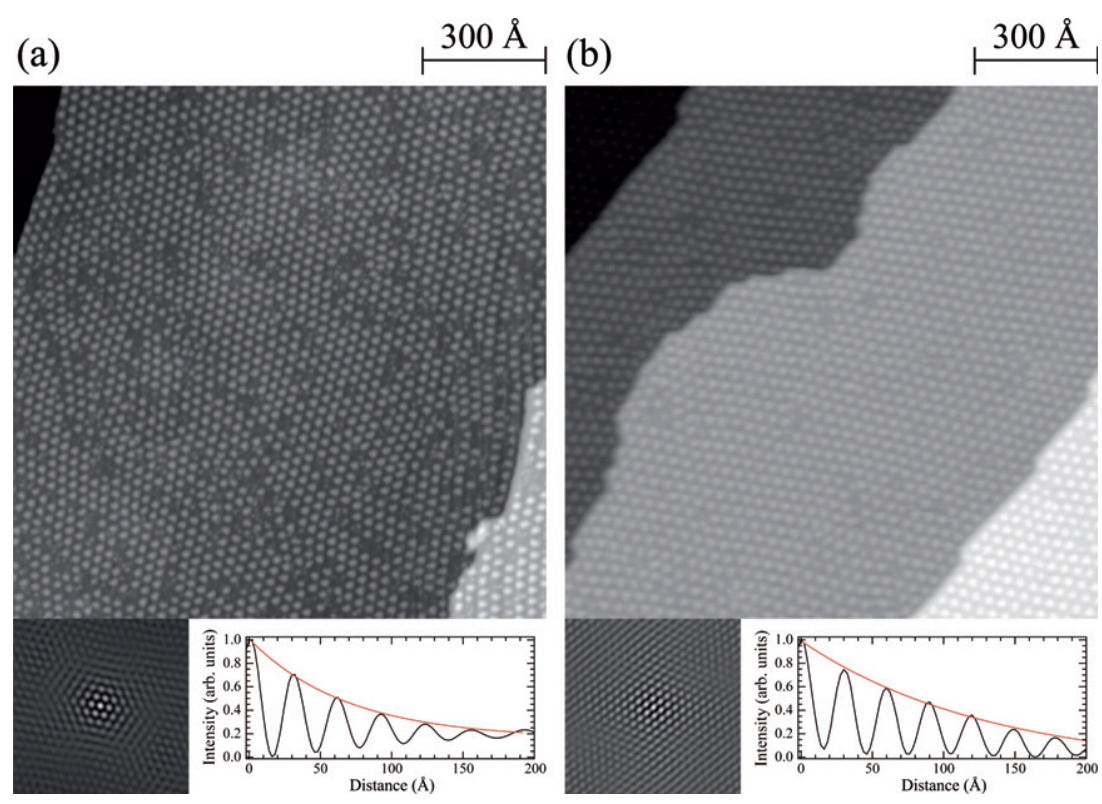

Figure 2. The temperature-dependent long-range order of graphene grown by ethylene CVD at (a) $T_{\mathrm{CVD}}=1100 \mathrm{~K}(20 \mathrm{~L})$ and (b) $T_{\mathrm{CVD}}=1670 \mathrm{~K}(801)$. After exposure the temperature is held for $2 \mathrm{~min}$ and then lowered to room temperature with $\dot{T}=-7 \mathrm{~K} \mathrm{~s}{ }^{-1}$. The lower left panels are 2D autocorrelation images and the lower right cuts through them show the exponential decay defining the autocorrelation length $\left(V_{\mathrm{t}}=-1.0 \mathrm{~V}, I_{\mathrm{t}}=316 \mathrm{pA}\right)$.
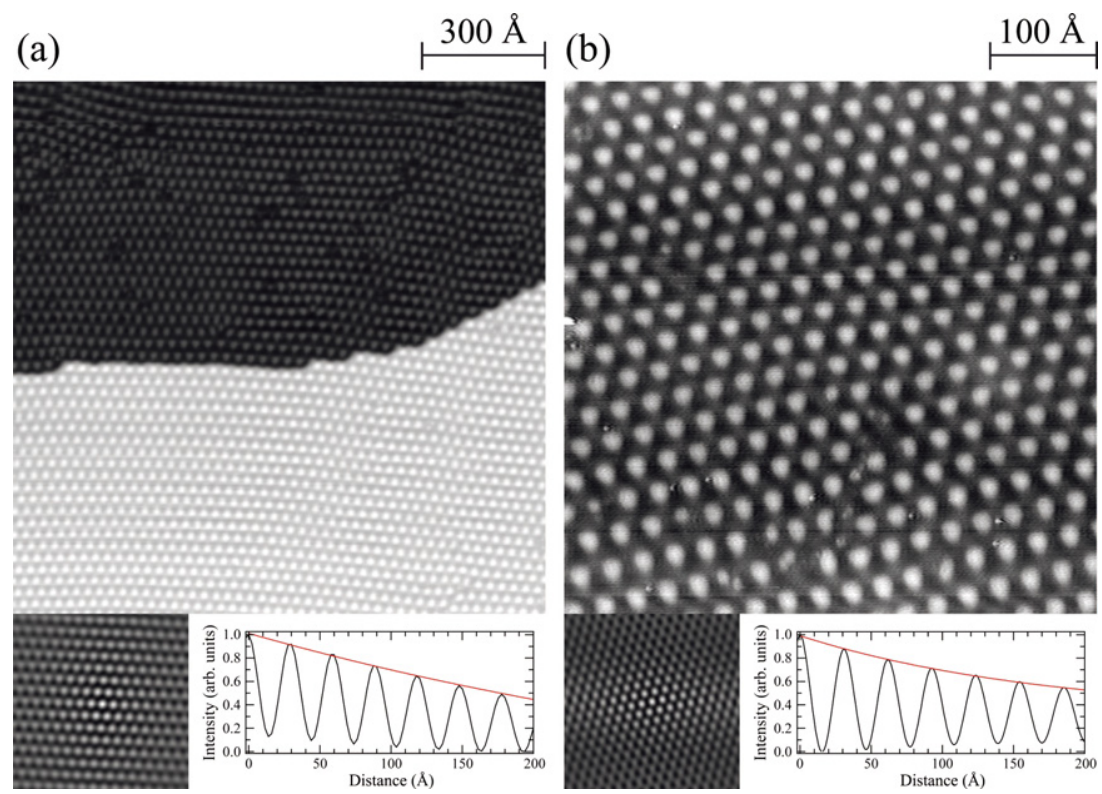

Figure 3. (a) The graphene layer with an autocorrelation length of $\Lambda=238 \pm 12 \AA$ obtained from intentionally dissolving carbon into the bulk during CVD and its subsequent controlled surface segregation during slow cooling $\left(127 \mathrm{~L} \mathrm{C}_{2} \mathrm{H}_{4}\right.$ at $T_{\mathrm{CVD}}=1540 \mathrm{~K}, \dot{T}=-0.1 \mathrm{~K} \mathrm{~s}{ }^{-1}$, $V_{\mathrm{t}}=-1 \mathrm{~V}, I_{\mathrm{t}}=316 \mathrm{pA}$ ). (b) Graphene growth during a short flash to $T=1570 \mathrm{~K}$ of a freshly prepared Ru surface due to surface segregation of the residual bulk dissolved carbon from previous graphene preparation cycles $\left(V_{\mathrm{t}}=-0.88 \mathrm{~V}, I_{\mathrm{t}}=150 \mathrm{pA}\right)$.

that a flash might reveal the preparation history of the $\mathrm{Ru}$ crystal. Such a sample is shown in figure 3(b) exhibiting with $\Lambda=(174 \pm 10) \AA$ very good long-range order. In table $1,{ }^{4}$ various preparation methods are compared with respect to

4 The literature autocorrelation lengths $(\Lambda)$ have been determined for the largest terraces in the STM images of the respective publication. The gray-scale has been used as the $z$-value and it has been verified with our own data that this makes no difference within the error bars with respect to taking the real $z$-values, even if non-linear gray-scale tables have been applied. their autocorrelation lengths for this and published work [13, $21,52]$. The best growth method for order is to combine CVD, carbon loading and segregation of the surface layer. This assessment can be rationalized as follows.

Concerted experimental $[14,17,23,24]$ and theoretical [54-56] effort has been devoted to addressing the graphene growth mechanism on transition metal surfaces. Once the thermal decomposition of the hydrocarbon molecule is complete, which happens at around $450 \mathrm{~K} \leq T \leq 700 \mathrm{~K}$ 
Table 1. Comparison of autocorrelation lengths $(\Lambda)$ obtained with different growth methods for graphene on $\mathrm{Ru}(0001)$ (see footnote 4).

\begin{tabular}{lll}
\hline Reference & $\Lambda(\AA)$ & Method \\
\hline$[13]$ & $(59 \pm 5)$ & TPG cycles \\
{$[21]$} & $(76 \pm 4)$ & Segregation \\
{$[52]$} & $(157 \pm 43)$ & CVD plus segregation \\
This work & $(68 \pm 12)$ & Low temperature CVD \\
This work & $(131 \pm 8)$ & High temperature CVD \\
This work & $(238 \pm 12)$ & CVD plus segregation \\
This work & $(174 \pm 10)$ & Long term preparation \\
\hline
\end{tabular}

[7, 8, 47], small carbon clusters reside on the surface. Only if the temperature is sufficiently high, $T \geq 700 \mathrm{~K}[7$, 47], will these carbon clusters start to form graphene islands [7, 47, 54, 55], given that a critical $\mathrm{C}$ adatom concentration is reached [17, 23, 24, 56]. Furthermore, it is now understood that graphene formation proceeds not via monomer attachment but rather through incorporation of a five carbon atom precursor molecule [17, 24, 56]. The stability of a graphene island is a complicated function of its size [55, 57]; particular cluster dimensions have an increased life-time [14] and exhibit varying electronic properties due to quantum confinement [58].

Interestingly, once larger graphene islands have nucleated, the structural quality of the resulting sheet is also determined by the speed of growth, i.e., the $\mathrm{C}$ adatom density [17, 23]. An example is shown in figure 4. This sample was prepared by keeping it for three more minutes after terminating the CVD growth at the growth temperature of $1100 \mathrm{~K}$. In agreement with figure 2(a), the center of the graphene patch is poorly ordered; however, it is bound by a very well ordered stripe of $40 \mathrm{~nm}$ width. The ethylene partial pressure gradually decreases after closing the leak-valve and so does the $\mathrm{C}$ adatom density. The resulting lower growth speed leads to higher graphene quality. In the end, it is the delicate balance between the $\mathrm{C}$ adatom density, the formation of precursor $\mathrm{C}$ clusters, their attachment to existing graphene patches, the purity of the $\mathrm{C}$ source, as well as the rearrangement between growing graphene islands upon coalescence that decides the resulting graphene quality [17, 56]. The superior structural quality of the third growth method (CVD plus segregation) can now be rationalized. On the one hand, it relies on the cleanliness of the approach, which avoids co-segregation of bulk dissolved impurities other than carbon. On the other hand, the low $\mathrm{C}$ supersaturation at high temperature leads to a slow carbon attachment to existing graphene patches with the concomitant excellent structural quality. We finally note that graphene formation may through the high growth temperatures also lead to substrate faceting $[59,60]$. Interestingly, the faceted regions provide especially well ordered graphene patches $[7,60]$, which could open a new route for more efficient growth of high quality graphene. The present results show the best long-range order for a combination of CVD growth and segregation.

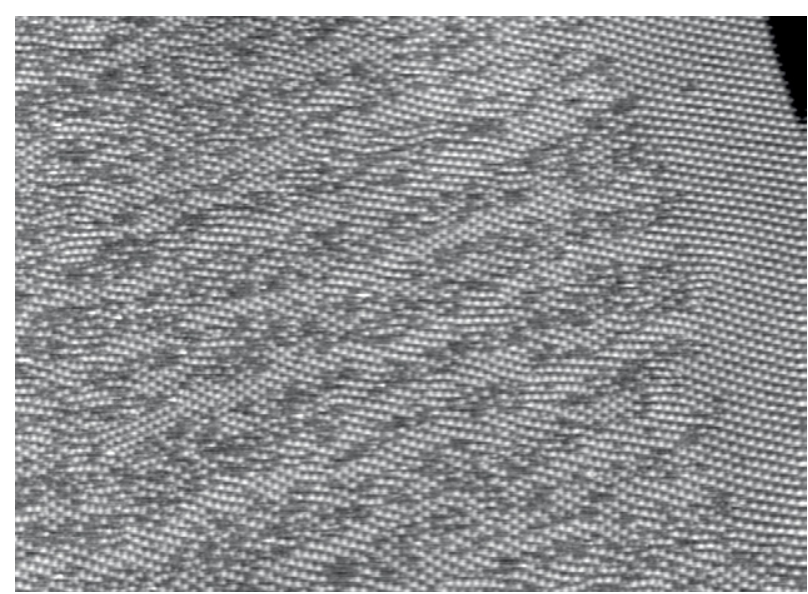

Figure 4. The ordered stripe at the border of a disordered graphene patch grown on $\mathrm{Ru}(0001)$ by ethylene $\mathrm{CVD}$ at low temperature with 3 min post-annealing $\left(1100 \mathrm{~K}, 20 \mathrm{~L}, p_{\mathrm{C}_{2} \mathrm{H}_{4}}=3.5 \times 10^{-7} \mathrm{mbar}\right.$, $\left.200 \times 146 \mathrm{~nm}^{2}, V_{\mathrm{t}}=-1.0 \mathrm{~V}, I_{\mathrm{t}}=150 \mathrm{pA}\right)$

\section{4. $g / \operatorname{Ir}(111)$}

We now describe the effect of a periodic potential on the electronic band structure of graphene resulting from the moiré structure of $\mathrm{g} / \operatorname{Ir}(111)-(9.25 \times 9.25)[12,15]$ and from its reinforcement by self-assembled Ir clusters grown on top [29]. For the first system mostly unperturbed Dirac cones at the K-points of the Brillouin zone, except for the opening of minigaps at the boundaries of the mini-Brillouin zone, have been reported [51]. Figure 5 shows STM images and ARPES intensities comparing both systems, revealing that the cluster superlattice potential induces a strong group velocity anisotropy together with a significant band gap opening [29]. We focus on the energy region close to the apex of the Dirac cone, and, since the linear dispersion of the $\pi$ band is modified close to the Bragg planes, we restrict our analysis to energies $E-E_{\mathrm{F}}>-0.5 \mathrm{eV}$. In the presence of the superlattice the group velocities in the two directions are $v_{\Gamma \mathrm{K}}=(4.90 \pm$ $0.06) \mathrm{eV} \AA$ and $v_{\mathrm{p} \Gamma \mathrm{K}}=(2.90 \pm 0.05) \mathrm{eV} \AA$, corresponding to an anisotropy of $\Delta v / v_{\mathrm{p} \Gamma \mathrm{K}}=(70 \pm 5) \%$. This value is 12 times larger than the anisotropy expected for unperturbed graphene due to its trigonal warping. The tight-binding approximation up to third nearest neighbors (TB3 model) for free-standing graphene gives $\Delta v / v_{\mathrm{p} \Gamma \mathrm{K}}=5 \%$ [61]. The anisotropy for $\mathrm{g} / \operatorname{Ir}(111)$ is, with $\Delta v / v_{\mathrm{p} \Gamma \mathrm{K}}=(16 \pm 2) \%$, larger than this, showing the small effect of the periodic potential resulting from the moiré alone. The group velocity renormalization is expected to be more effective for charge carriers moving perpendicular to the largest corrugation of the potential [26, 27]. In agreement, we find the strongest reduction for $\mathrm{p} \Gamma \mathrm{K}$.

The superlattice induced band gap opening can be derived by comparing the positions of the Dirac cone summits for both cases, and by making reasonable assumptions on the position of the Dirac point derived by carefully checking for charge neutrality. For $\mathrm{g} / \mathrm{Ir}$ we find $E_{\pi}=(-70 \pm 20) \mathrm{meV}$, in good agreement with previous results [35]. The Ir cluster superlattice shifts the $\pi$ summit down to $E_{\pi}=(-200 \pm$ 20) $\mathrm{meV}$, while the $\pi^{*}$ band stays above $E_{\mathrm{F}}$. Following the 
(a)

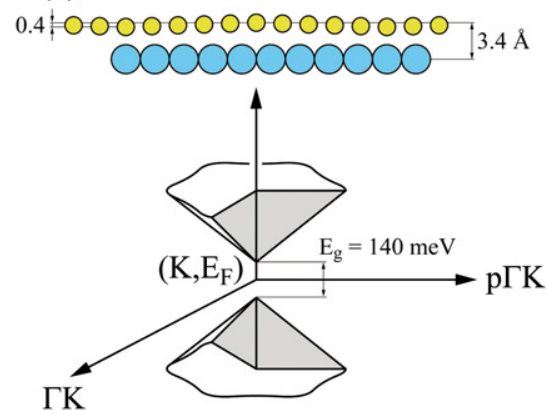

(b)

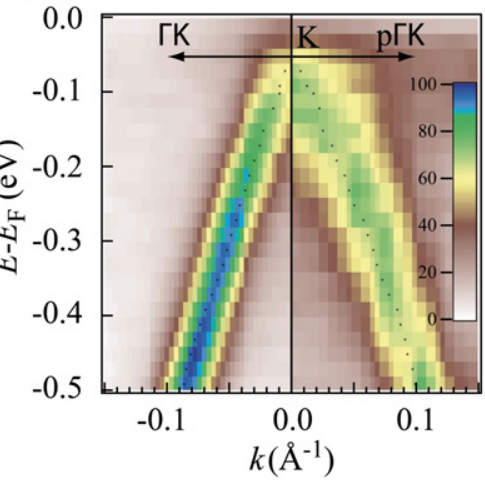

(d)

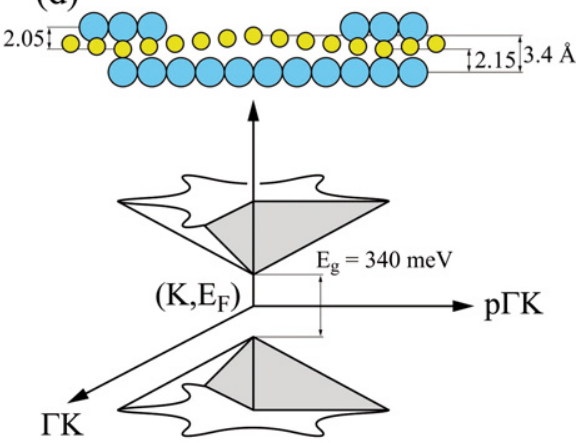

(e)
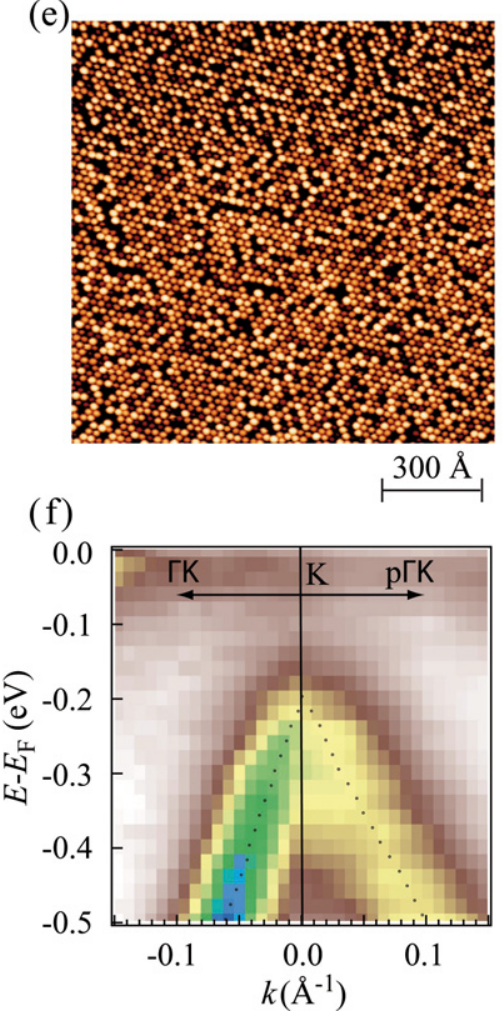

Figure 5. (a) Sketch of the $\pi\left(\pi^{*}\right)$ band for $\mathrm{g} / \mathrm{Ir}(111)$ close to the K-point and $E_{\mathrm{F}}$. The Dirac cones are almost symmetric and only a small gap opens at the K-point. (b) STM image of the moiré structure of $g / \operatorname{Ir}(111)$. (c) ARPES intensity of the $\pi$ band of $g / \operatorname{Ir}(111)$ around the K-point and along the $\Gamma$ K direction and perpendicular to it. (d) Sketch of the $\pi\left(\pi^{*}\right)$ band for $\operatorname{Ir} / \mathrm{g} / \operatorname{Ir}(111)$. The cluster superlattice induces a strong anisotropy in the Dirac cones and the opening of a large gap at the K-point. (e) STM image of an Ir cluster superlattice grown on $\mathrm{g} / \operatorname{Ir}(111)\left(\theta=0.15 \mathrm{ML}, T_{\mathrm{dep}}=375 \mathrm{~K}\right)$. (f) ARPES intensity of the $\pi$ band for $\operatorname{Ir} / \mathrm{g} / \mathrm{Ir}(111)$ around the K-point and along the $\Gamma \mathrm{K}$ direction and perpendicular to it. In (c) and (f) the dots represent the peak positions of the momentum distribution curves.

literature [28, 35], we assume charge neutrality for $\mathrm{g} / \mathrm{Ir}(111)$ and find $E_{\mathrm{g}, \mathrm{g} / \mathrm{Ir}}=(140 \pm 40) \mathrm{meV}$. For the cluster lattice a possible charge transfer can be estimated from the core level and conduction band shifts. We find that these shifts are very small. The C $1 \mathrm{~s}$ level shifts down by $(30 \pm 30) \mathrm{meV}$ and the top of the $\sigma$ band at the M-point of the second Brillouin zone by the same amount. With $E_{\mathrm{D}}=-30 \mathrm{meV}$ we find $E_{\mathrm{g}, \mathrm{Ir} / \mathrm{g} / \mathrm{Ir}}=340 \mathrm{meV}$.

Further tuning of the graphene electronic bands can be achieved by chemisorption of alkali metals at room temperature. For $\mathrm{Na}$ adsorption on bare graphene $(\mathrm{Na} / \mathrm{g})$ we observe a band gap of $E_{\mathrm{g}, \mathrm{Na} / \mathrm{g} / \mathrm{Ir}}=320 \mathrm{meV}$, whereas in the case of $\mathrm{Na}$ adsorption on the Ir cluster superlattice the band gap reaches $E_{\mathrm{g}, \mathrm{Na} / \mathrm{Ir} / \mathrm{g} / \mathrm{Ir}}=740 \mathrm{meV}$ (see figure 6(a)) [42], which is very close to the band gap of conventional semiconductors. Remarkably, the formation of these very large band gaps preserves the shape of the Dirac cone and the high electron group velocity. Due to strong electron charge transfer from Na to graphene, the $\pi^{*}$ state is shifted below $E_{\mathrm{F}}$ as compared with bare graphene on $\operatorname{Ir}(111)$. From the constant $\mathrm{d} E / \mathrm{d} k$ gradient of the band dispersion of the $\pi^{*}$ states we evaluate the electron group velocities to $v_{\mathrm{Na} / \mathrm{g} / \mathrm{Ir}} \approx 5.5 \mathrm{eV} \AA$ and $v_{\mathrm{Na} / \mathrm{Ir} / \mathrm{g} / \mathrm{Ir}} \approx 5.2 \mathrm{eV} \AA$, respectively. These values are very close to the ones obtained by third nearest neighbor tight-binding calculations [61] and in magneto-transport measurements of free-standing graphene.

Figure 6(b) shows a schematic summary of the evolution of the graphene band gap as a function of the applied 


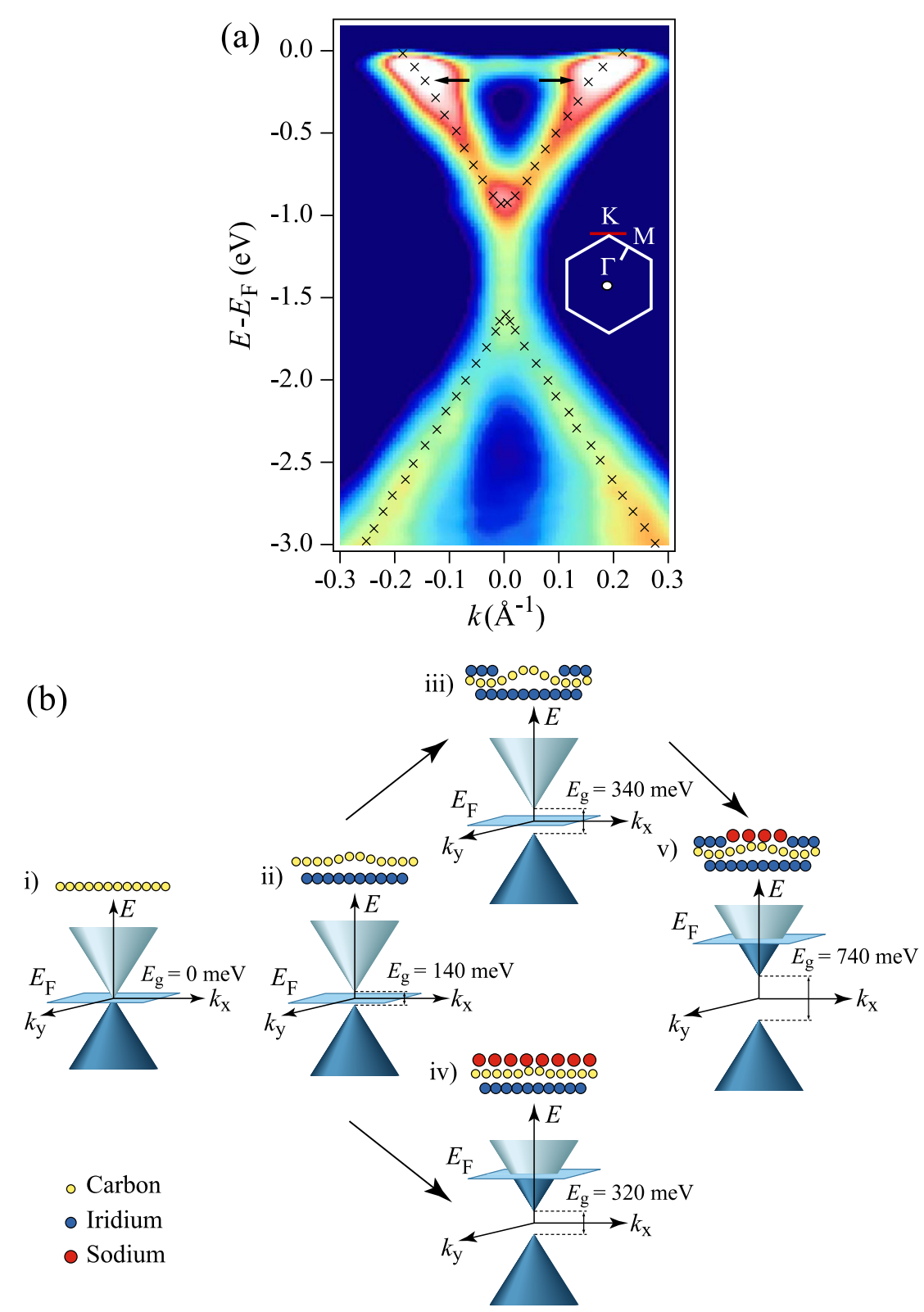

Figure 6. (a) ARPES map along the $\mathrm{p} \Gamma \mathrm{K}$ direction for $\mathrm{Na} / \mathrm{Ir} / \mathrm{g} / \mathrm{Ir}(111)$. The inset shows the $2 \mathrm{D}$ Brillouin zone of graphene. The red line indicates the $\mathrm{p} \Gamma \mathrm{K}$ direction. The black crosses overlaid on the ARPES maps mark the maximum of the photoemission spectral-weight intensity whereas the arrows highlight kinks in the electronic band dispersion generated by the electron-phonon interaction. (b) Side view sketch of the atomic structure and of the $\pi$ and $\pi^{*}$ bands close to the K-point of the graphene Brillouin zone and at $E_{\mathrm{F}}$ for (i) free-standing graphene, (ii) g/Ir, (iii) Ir/g/Ir, (iv) $\mathrm{Na} / \mathrm{g} / \mathrm{Ir}$ and (v) Na/Ir/g/Ir.

perturbation. In order to give a possible explanation for the $\mathrm{Na}$ induced band gap we note that the STM data show the adsorption of $\mathrm{Na}$ on top of graphene, most likely in a $(2 \times 2)$ phase, and rule out intercalation at room temperature [42]. Although a $(2 \times 2)$ reconstruction is not expected to remove the degeneracy between graphene states at the K-point, a band gap may be induced by hybridization between the electron wavefunctions with wavevectors $K$ and $K^{\prime}$ promoted by the translation symmetry breaking of the combined $\mathrm{Na}$-graphene- $\operatorname{Ir}(111)$ lattice. We attribute the wide gap to $\mathrm{Na}$ and Ir jointly breaking the degeneracy of the two graphene sublattices.

\section{Conclusions}

We have reviewed and presented growth techniques for graphene on $\mathrm{Ru}(0001)$ leading to large autocorrelation lengths of up to $240 \AA$. The electronic properties of graphene on $\operatorname{Ir}(111)$ could be tailored while preserving high charge carrier group velocities, a necessary condition for high mobilities.

\section{Acknowledgments}

We gratefully acknowledge the contributions of $\mathrm{P}$ Moras, S Vlaic, M Etzkorn, P M Sheverdyaeva, and D Pacilé to 
this work. We acknowledge support from the Swiss National Science Foundation, from the European Science Foundation under the EUROCORES Program EuroGRAPHENE, and from the Italian Ministry of Education, University and Research (FIRB Futuro in Ricerca, PLASMOGRAPH Project).

\section{References}

[1] Lin Y M, Dimitrakopoulos C, Jenkins K A, Farmer D B, Chiu H Y, Grill A and Avouris P 2010 Science 327662

[2] Chen J-H, Cullen W G, Jang C, Fuhrer M S and Williams E D 2009 Phys. Rev. Lett. 102236805

[3] Li X et al 2009 Science 3241312

[4] Bae S et al 2010 Nature Nanotechnol. 5574

[5] Iwasaki T, Park H J, Konuma M, Lee D S, Smet J H and Starke U 2011 Nano Lett. 1179

[6] Novoselov K S, Geim A K, Morozov S V, Jiang D, Zhang Y, Dubonos S V, Grigorieva I V and Firsov A A 2004 Science 306666

[7] Baron K, Blakely D and Somorjai G 1974 Surf. Sci. 4145

[8] Nieuwenhuys B, Hagen D, Rovida G and Somorjai G 1976 Surf. Sci. 59155

[9] Hrbek J 1986 J. Vac. Sci. Technol. A 486

[10] Land T A, Michely T, Behm R J, Hemminger J C and Comsa G 1991 Appl. Phys. A 53414

[11] Livneh T and Asscher M 2000 J. Phys. Chem. B 1043355

[12] N'Diaye A T, Bleikamp S, Feibelman P J and Michely T 2006 Phys. Rev. Lett. 97215501

[13] Vazquez de Parga A L, Calleja F, Borca B, Passeggi M C G, Hinarejos J J, Guinea F and Miranda R 2008 Phys. Rev. Lett. 100056807

[14] Wang B, Ma X, Caffio M, Schaub R and Li W-X 2011 Nano Lett. 11424

[15] Coraux J, N'Diaye A T, Busse C and Michely T 2008 Nano Lett. 8565

[16] N'Diaye A T, Coraux J, Plasa T N, Busse C and Michely T 2008 New J. Phys. 10043033

[17] Loginova E, Bartelt N C, Feibelman P J and McCarty K F 2009 New J. Phys. 11063046

[18] Yoshii S, Nozawa K, Toyoda K, Matsukawa N, Odagawa A and Tsujimura A 2011 Nano Lett. 112628

[19] Arnoult W J and McLellan R B 1972 Scr. Metall. 61013

[20] Weiss N 2004 PhD Thesis EPFL

[21] Marchini S, Günther S and Wintterlin J 2007 Phys. Rev. B 76075429

[22] Sutter P W, Flege J-I and Sutter E A 2008 Nature Mater. 7406

[23] McCarty K F, Feibelman P J, Loginova E and Bartelt N C 2009 Carbon 471806

[24] Loginova E, Bartelt N C, Feibelman P J and McCarty K F 2008 New J. Phys. 10093026

[25] Natterer F D, Rusponi S and Brune H 2011 SPG Mitt. 3431

[26] Park C H, Son Y W, Yang L, Cohen M L and Louie S G 2009 Phys. Rev. Lett. 103046808

[27] Brey L and Fertig H A 2009 Phys. Rev. Lett. 103046809

[28] Feibelman P J 2008 Phys. Rev. B 77165419

[29] Rusponi S, Papagno M, Moras P, Vlaic S, Etzkorn M, Sheverdyaeva P M, Pacile D, Brune H and Carbone C 2010 Phys. Rev. Lett. 105246803

[30] Yang L, Park C H, Son Y W, Cohen M L and Louie S G 2007 Phys. Rev. Lett. 99186801
[31] Cai J et al 2010 Nature 466470

[32] Bai J, Zhong X, Jiang S, Huang Y and Duan X 2010 Nature Nanothecnol. 5190

[33] Liang X, Jung Y-S, Wu S, Ismach A, Olynick D L, Cabrini S and Borok J 2010 Nano Lett. 102454

[34] Yavari F, Kritzinger C, Gaire C, Song L, Gulapalli H, Borca-Tasciuc T, Ajayan P M and Koratkar N 2010 Small 62535

[35] Balog R et al 2010 Nature Mater. 9315

[36] Starodub E, Bostwick A, Moreschini L, Nie S, Gabaly F E, McCarty K F and Rotenberg E 2011 Phys. Rev. B 83125428

[37] Varykhalov A, Scholz M R, Kim T K and Rader O 2010 Phys. Rev. B 82 121101(R)

[38] Haberer D et al 2010 Nano Lett. 103360

[39] Guinea F, Katsnelson M I and Geim A K 2010 Nature Phys. 630

[40] Ohta T, Bostwick A, Seyller T, Horn K and Rotenberg E 2006 Science 313951

[41] Zhou S Y, Gweon G H, Fedorov A V, First P N, Heer W A D, Lee D H, Guinea F, Neto A H C and Lanzara A 2007 Nature Mater. 6770

[42] Papagno M, Rusponi S, Sheverdyaeva P M, Vlaic S, Etzkorn M, Pacilé D, Moras P, Carbone C and Brune H 2012 ACS Nano 6199

[43] Lehnert A, Buluschek P, Weiss N, Giesecke J, Treier M, Rusponi S and Brune H 2009 Rev. Sci. Instrum. 80023902

[44] Martoccia D et al 2008 Phys. Rev. Lett. 101126102

[45] Martoccia D, Björck M, Schlepütz C M, Brugger T, Pauli S A, Patterson B D, Greber T and Willmott P R 2010 New J. Phys. 12043028

[46] Moritz W, Wang B, Bocquet M L, Brugger T, Greber T, Wintterlin J and Günther S 2010 Phys. Rev. Lett. 104136102

[47] Land T A, Michely T, Behm R J, Hemminger J C and Comsa G 1992 Surf. Sci. 264261

[48] Griffiths K, Lennard W, Mitchell I, Norton P, Pirug G and Bonzel H 1993 Surf. Sci. Lett. 284 L389

[49] Wang B, Bocquet M-L, Marchini S, Gunther S and Wintterlin J 2008 Phys. Chem. Chem. Phys. 103530

[50] Iannuzzi M and Hutter J 2011 Surf. Sci. 6051360

[51] Pletikosic I, Kralj M, Pervan P, Brako R, Coraux J, N'Diaye A T, Busse C and Michely T 2009 Phys. Rev. Lett. 102056808

[52] Sutter E, Acharya D P, Sadowski J T and Sutter P 2009 Appl. Phys. Lett. 94133101

[53] Banhart F, Kotakoski J and Krasheninnikov A V 2011 ACS Nano $\mathbf{5} 26$

[54] Chen H, Zhu W and Zhang Z 2010 Phys. Rev. Lett. 104186101

[55] Gao J, Yuan Q, Hu H, Zhao J and Ding F 2011 J. Phys. Chem. C 11517695

[56] Zangwill A and Vvedensky D D 2011 Nano Lett. 112092

[57] Yuan Q, Gao J, Shu H, Zhao J, Chen X and Ding F 2012 J. Am. Chem. Soc. 1342970

[58] Phark S H, Borme J, Vanegas A L, Corbetta M, Sander D and Kirschner J 2011 ACS Nano 58162

[59] Starodub E, Maier S, Stass I, Bartelt N C, Feibelman P J, Salmeron M and McCarty K F 2009 Phys. Rev. B 80235422

[60] Günther S, Dünhardt S, Wang B, Bocquet M-L, Schmitt S and Wintterlin J 2011 Nano Lett. 111895

[61] Reich S, Maultzsch J, Thomsen C and Ordejón P 2002 Phys. Rev. B 66035412 